Provided for non-commercial research and education use. Not for reproduction, distribution or commercial use.

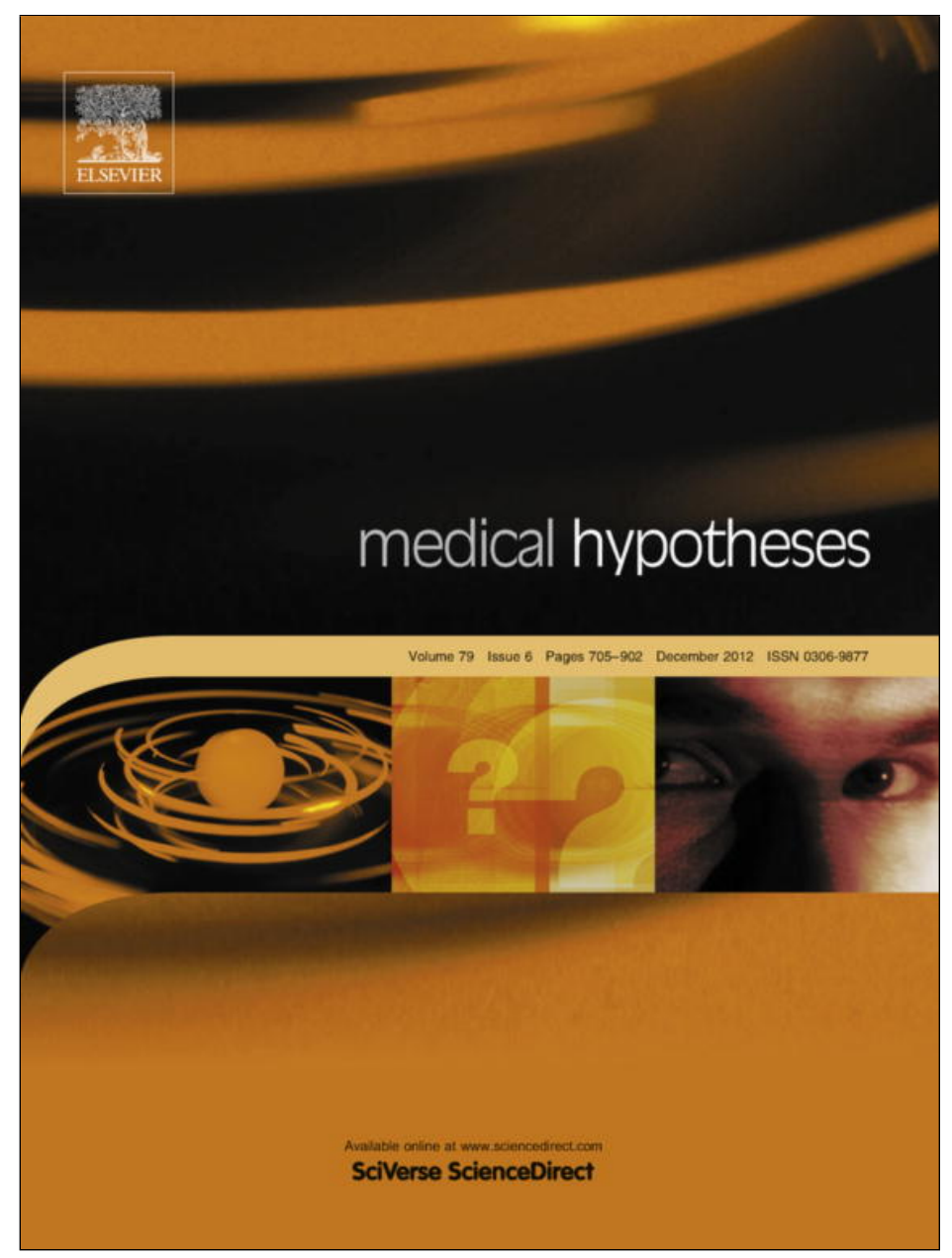

This article appeared in a journal published by Elsevier. The attached copy is furnished to the author for internal non-commercial research and education use, including for instruction at the authors institution and sharing with colleagues.

Other uses, including reproduction and distribution, or selling or licensing copies, or posting to personal, institutional or third party websites are prohibited.

In most cases authors are permitted to post their version of the article (e.g. in Word or Tex form) to their personal website or institutional repository. Authors requiring further information regarding Elsevier's archiving and manuscript policies are encouraged to visit:

http://www.elsevier.com/copyright 


\title{
Dissecting the different biological effects of oncogenic Ras isoforms in cancer cell lines: Could stimulation of oxidative stress be the one more weapon of H-Ras? Regulation of oxidative stress and Ras biological effects
}

\author{
Maurizio Bellavia ${ }^{\mathrm{a}, \mathrm{b}, *, 1}$, Maria Concetta Gioviale ${ }^{\mathrm{b}}$, Giuseppe Damiano ${ }^{\mathrm{b}}$, Vincenzo Davide Palumbo ${ }^{\mathrm{b}}$, \\ Gabriele Spinelli ${ }^{\mathrm{b}}$, Giuseppe Buscemi ${ }^{\mathrm{a}, \mathrm{b}}$, Attilio Ignazio Lo Monte ${ }^{\mathrm{a}, \mathrm{b}}$ \\ a Department of Surgical and Oncological Disciplines, University of Palermo, Palermo, Italy \\ ${ }^{\mathrm{b}}$ University Hospital of Palermo, School of Medicine, Palermo, Italy
}

\section{A R T I C L E I N F O}

\section{Article history:}

Received 28 September 2011

Accepted 15 August 2012

\begin{abstract}
A B S T R A C T
Ras proteins are small GTPase functioning as molecular switches that, in response to particular extracellular signalling, as growth factors, activate a diverse array of intracellular effector cascades regulating cell proliferation, differentiation and apoptosis. Human tumours frequently express Ras proteins (Ha-, Ki-, NRas) activated by point mutations which contribute to malignant phenotype, including invasiveness and angiogenesis. Despite the common signalling pathways leading to similar cellular responses, studies clearly demonstrate unique roles of the Ras family members in normal and pathological conditions and the lack of functional redundancy seems to be explainable, at least in part, by the ability of Ras isoforms to localize in different microdomains to plasma membrane and intracellular organelles. This different intracellular compartmentalization could help Ras isoforms to contact different downstream effectors finally leading to different biological outcomes.

Interestingly, it has also been shown that Ha- and Ki-Ras exert an opposite role in regulating intracellular redox status.

In this regard we suggest that H-Ras specific induction of ROS (reactive oxygen species) production could be one of the main determinants of the invasive phenotype which characterize cancer cells harbouring H-Ras mutations. In our hypothesis then, while K-Ras (not able to promote oxidative stress) could mainly contribute to cancer progression and invasiveness through activation of MAPK and PI3 K, $\mathrm{H}$-Ras-mediated oxidative stress could play a unique role in modulation of intercellular contacts leading to a loss of cell adhesion and eventually also to a metastatic spread.
\end{abstract}

(c) 2012 Elsevier Ltd. All rights reserved.

\section{Introduction}

Three closely related Ras isoforms, Harvey rat sarcoma viral oncogene homolog (termed H-Ras), neuroblastoma Ras viral oncogene homolog (termed N-Ras) and Kirsten rat sarcoma viral oncogene homolog (termed K-Ras), have sequence homology between residues 1 and 165 corresponding to GDP-GTP binding and effectors interactions motifs. The portion of the sequence which differs in the three different Ras isoforms is the C-terminal hypervariable

\footnotetext{
* Corresponding author. Address: Research Fellow at the University of Palermo, Department of Surgical and Oncological Disciplines, School of Medicine-School of Biotechnology, Via del Vespro, 129, 90127 Palermo, Italy. Tel.: +39 09165526 66, mobile: +39 32070279 87; fax: +39 0916552634

E-mail addresses: mauriziobellavia1@hotmail.it, mauriziobellavia1@virgilio.it (M. Bellavia).

${ }^{1} \mathrm{PhD}$ in Experimental Oncobiology, Research Fellow at the University of Palermo, Palermo, Italy.
}

region (HVR), which contains the major motifs for membrane attachment and targeting to distinct intracellular compartments. After synthesis on cytosolic polysomes, Ras isoforms undergo post-translation modifications which affect their membrane affinity [1]. In particular the lack of hydrophobic acyl groups on K-Ras facilitates its shuttling between the cell surface and intracellular organelles [2]; interestingly K-Ras can migrate to mitochondria and regulate apoptosis [3]. On the other hand palmitoyl groups on H-Ras make it able to localize preferentially to the lipid rafts [4].

Ras proteins (comprising Ha-Ras, Ki-Ras and N-Ras) activate different signal pathways responsible for control of proliferation, cell adhesion, apoptosis and cell migration.

Mutations in the Ras family of proto-oncogenes are found in $30 \%$ of all human tumours and in $50 \%$ of colon tumours in particular [5]. The point mutations affect usually residues 12 and 61; in particular the glycine to valine mutation on residue 12 renders Ras insensitive to inactivation leading to increased invasion and metastasis and decreased apoptosis. 
Ras proteins modulate the activity of several downstream molecular effectors, among them the mitogen activated protein (MAP) kinases and PI3 K are the most studied [6,7]. Despite their high degree of sequence homology Ha-, Ki- and N-Ras proteins exert different biochemical activities [8]. For example it has been shown a different affinity for downstream molecular effectors as Raf and phosphoinositide 3-kinase (PI3K) and a unique role of K-Ras during mouse development [9-11].

Interestingly, published data show also that H-Ras can induce NADPH-oxidase (Nox) system through activation of ERK 1/2 leading to an increase in intracellular ROS levels [12-15] while K-Ras, on the other hand, can determine a resistance to oxidative stress [16] and it can stimulate the scavenging of ROS by activating posttranscriptionally the mitochondrial antioxidant enzyme Mn-superoxide dismutase (Mn-SOD) in a ERK 1/2 dependent manner $[17,15]$.

\section{Hypothesis}

Reactive oxygen species (ROS) exert a role in tumour metastasis; particularly they are involved in epithelial-mesenchymal transition (EMT), migration, invasion of the tumour cells and angiogenesis [18-21]. These biological effects are mediated through activation of MAPK which stimulate several transcriptional factors controlling cancer-associated cellular behaviour: activation of Smad determines, for example, a decrease in cell adhesion and an increase in migration and invasion, and activation of Snail causes a change in cellular shape, referred as epithelial mesenchymal transition, which is associated with acquisition of motility and loss of cell adhesion too [22].

The membrane-associated NADPH oxidase system (Nox1), together with mitochondria, is one of the main sources of ROS within the cell and its biochemical activity is able to determine a persistent directed cellular migration through exportation of $\alpha 2 \beta 1$ integrins to cell surface [23]; it has also been proposed that Nox1 could be a target for cancer therapy [24]. Intriguing published data showed that NADPH oxidase-generated ROS are required for the formation and function of invadopodia, which are cellular structures required for cellular motility and invasivity; in particular in a mouse fibrosarcoma model system (Src -3T3 cells) Nox1-derived ROS showed the ability to stimulate phosphorilation of Tks4 and Tks5 [25], which are two proteins exerting a main role in the function of invadopodia [26,27].

Also ROS of mitochondrial origin are related to acquisition of an invasive phenotype through activation of Ets- 1 transcription factor which determines expression of metalloproteinase or through down regulation of metalloproteinase inhibitors [28,29].

In favour of a crucial role of ROS in cancer metastasis, it has also been shown, in animal models, that targeted delivery of catalase, an enzyme counteracting ROS-related oxidative stress, was able to inhibit tumour metastasis to the liver, lung and peritoneal organs [30].

We suggest that H-Ras mediated production of ROS, through activation of Nox system, could be a peculiar molecular mechanism by which this specific isoform of the Ras family proteins mediate the process of cancer cell migration and invasion, leading to metastasis; K-Ras, on the other hand, could be able to stimulate invasion and migration, through other molecular effectors, probably shared with H-Ras, as PI3K [31]

\section{Evaluation of hypothesis and discussion}

$\mathrm{H}-$ and K-Ras are two isoforms of the Ras family proteins but they have different biological effects. In particular it has been shown that H-Ras is frequently associated to a more aggressive and invasive phenotype because it is specifically able to activate Rac-MKK3/6-p38 pathway [32] and it could increase MMP-2 [33], urokinase [34], MMP-9 and COX-2 [35], Fyn [36] expression, while K-Ras has been proposed to play a unique role in initiation of tumours of endodermal origin via stem cell expansion [37]; interestingly in the latter case H-Ras induced, on the contrary, differentiation and growth arrest of these cells. The work by Quinlan and co-workers [37] seem then point out a specific role of K-Ras in tumour initiation, while $\mathrm{H}$-Ras could exert an action at a later phase of cancer progression inducing metastasis formation.

In this regard, as aforementioned, we speculate that $\mathrm{H}$-Ras could induce metastasis of cancer cells through its unique ability to determine ROS production via activation of the Nox system; in favour of this argument published data showed that pharmacological inhibition of this enzymatic complex suppressed MMP-13 (collagenase 3) expression mediated by H-Ras [38] and that H-Ras overexpression enhanced NF-kB binding to motifs in the MMP-9 gene promoter by increasing cellular superoxide production [39]. Interestingly, it has also been proposed that H-Ras-mediated production of ROS could be sufficient to induce transformation of transfected rat kidney epithelial cells; furthermore in vivo studies of tumour formation in nude mice showed that overexpression of glutathione peroxidase 1 , an antioxidant enzyme, and completely suppressed tumour cell growth [40]. Finally, the evidence that ROS can also stabilize H-Ras protein inhibiting its proteasomal degradation [41] lead us to speculate that the protein, inducing ROS production, could activate a self-stimulating mechanism which probably causes an increase in intensity and duration of the oncogenic signal. In this regard it is worthy of mention also the ability of ROS to inhibit protein tyrosine posphatases $[42,43]$ which are negative regulator of receptor tyrosine kinase (RTK); given that Ras is usually involved in signal transduction downstream RTK interaction with growth factors, H-Ras-mediated increase in intracellular ROS could contribute to amplify growth factor signalling.

ROS and H-Ras seem then act synergistically determining an autocatalytic cycle which could cause a drastic increase both in intensity and duration of signal; in our knowledge K-Ras, on the other hand, is not able to determine such an autocatalytic cycle of signalling activation. Furthermore K-Ras, through activation of MnSOD and catalase, counteract the metastasis-inducing effect of mitochondrial ROS (Fig. 1).

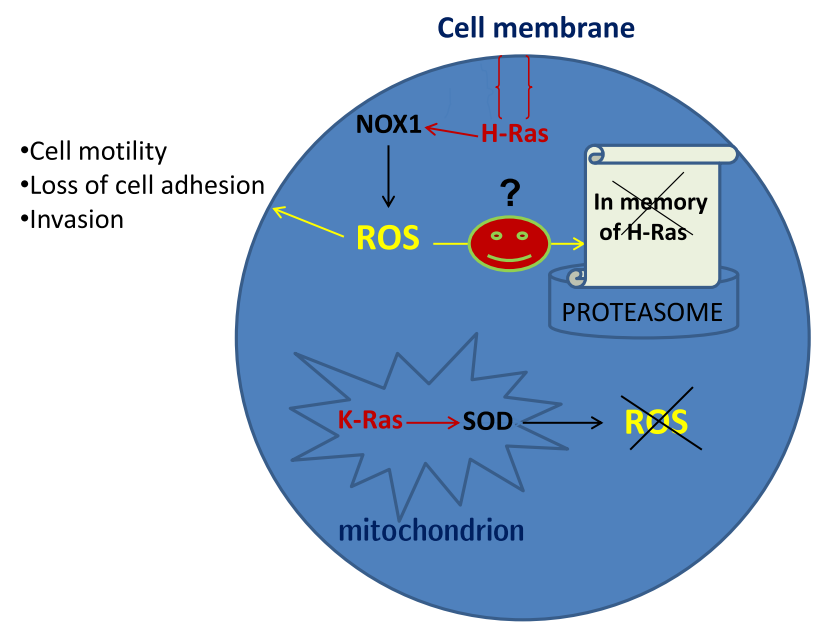

Fig. 1. Different pathways triggered by $\mathrm{H}-$ and $\mathrm{K}-\mathrm{Ras}$ in regulation of ROS production. H-Ras, localizing in cellular membrane microdomains close to Nox1 activates it leading to ROS production and biological effects as cell motility, loss of cell adhesion and invasion. ROS may also, through an unknown mechanism, counteract proteasome-mediated H-Ras proteolytic degradation. K-Ras, on the other hand, being able to localize inside mitochondria, positively regulate SOD gene expression ultimately causing a fall in intracellular ROS levels. 
Both H- and K-Ras exert their opposite role in regulating intracellular redox status through activation of ERK 1/2 downstream effectors; this seems a paradox, because it is difficult to understand how the same pathway could determine such an opposite biological outcome. In this regard a reasonable explanation appears the aforementioned ability of the two Ras isoforms to localize in different membrane microdomains. The H-Ras signalling complex, enucleating in the lipid rafts [4], could activate ERK 1/2 contiguous to NADH oxidase complex [44-46]. Furthermore it has been shown that Rac1, which is induced by H-Ras [47], is sufficient to stimulate the NADH oxidase complex assembly [48]; H-Ras could then also activate Nox1 in a Rac1-dependent, ERK 1/2 independent manner.

K-Ras, on the other hand, being able to migrate to mitochondria [3], could activate the mitochondrial antioxidant enzyme MnSOD; interestingly Bivona and co-workers [3] showed that mitochondrial localization of K-Ras depends on phosphorilation on a serine 181 within a polybasic region lacking in H-Ras. From this point of view the specific ability of K-Ras to stimulate activity of a mitochondrial enzyme (MnSOD) could be explained again, as for H-Ras and NADPH oxidase, by the correlation between protein sequence and specific intracellular localization. In this regard it seems very interesting that a glutamic acid substitution of the four lysine residues at the polybasic stretch of the $\mathrm{COOH}$ terminus of K-Ras, the region also crucial for mitochondrial translocation, completely abolished the post-transcriptional activation of MnSOD [17].

\section{Conclusions and future perspectives}

In this paper we speculate about the possible crucial role of ROS in mediating a cellular behaviour similar to metastatization; H-Ras could have a very important role in stimulating such process being necessary to "maintain" tumorigenicity while K-Ras, on the other hand, could be necessary to trigger the early steps of neoplastic transformation, even if it is involved in cancer cells invasion too.

Ras proteins localize to the plasma membrane where they function as molecular switches activating signal transduction pathways; this localisation depends on complex post-translational processing which increase proteins hydrophobicity. The crucial post-translational modification, shared by all Ras isoforms, consists of a prenylation catalysed by the enzyme farnesyltransferase (PFTase) and this is the reason why one of the promising molecular therapeutically approaches in the treatment of cancer patients could be administration of PFTase inhibitor [49]; despite all this therapy is no specific because it is directed against all Ras isoforms.

Interestingly, it has been recently reported that hypervariable region (HVR), consisting of amino acids 166-189 in H-Ras, determines the invasive/migratory signalling program [50].

We suggest that an "intelligent anticancer therapy" could be directed against this specific motif of Ha-Ras protein or, alternatively, against the motif, once identified, which is responsible of the specific activation of Nox system; this could help to drastically reduce intracellular level of ROS in cancer cells harbouring an activated $\mathrm{H}$-Ras protein, preventing acquisition of invasive and migratory phenotype. In our knowledge there are no experimental data regarding knockdown or silencing of H-Ras in cancer cell lines, while such experiments were carried out for K-Ras isoform. In particular, in human lung adenocarcinoma cells, it was showed that adenovirus-mediated siRNA suppression of activated K-Ras gene expression caused inhibition of in vitro and in vivo cell growth [51] and in human pancreatic cancer cells silencing of mutant K-Ras disrupted the malignant phenotype of pancreatic adenocarcinoma cells [52].

ROS, when present at an high intracellular level, are also well known mediators of cell death so an alternative ROS-based anticancer therapy could be the stimulation of their production to cause apoptosis in cancer cells; such therapeutic strategy should be oriented, in a high specific manner, only towards cancer cells but, in our opinion, it seems very difficult to achieve.

\section{Conflict of interest statement}

All authors declare the absence of any employment, consultancies, stock ownership, honoraria, paid expert testimony, patent applications/registrations, and grants which could inappropriately influence their work.

\section{References}

[1] Omerovic J, Prior IA. Compartmentalized signalling: Ras proteins and signalling nanoclusters. FEBS J 2009;276:1817-25.

[2] Plowman SJ, Ariotti N, Goodall A, Hancock JF. Electrostatic interaction positively regulate K-Ras nanocluster formation and function. Mol Cell Biol 2008;28:4377-85.

[3] Bivona TG, Quatela SE, Bodemann BO, et al. PKC regulates a farnesylelectrostatic switch on K-Ras that promotes its association with Bcl-XL on mitochondria and induces apoptosis. Mol Cell 2006;21:481-93.

[4] Prior IA, Harding A, Yan J, et al. GTP-dependent segregation of H-Ras from lipid rafts is required for biological activity. Nat Cell Biol 2001;3:368-75.

[5] Bos JL. Ras oncogenes in human cancer: a review. Cancer Res 1989;49:4682-9.

[6] Feig LA, Buchsbaum RJ. Cell signalling: life or death decisions of Ras proteins. Curr Biol 2002;12:R259-61.

[7] Mendoza MC, Er EE, Blenis J. The Ras-ERK and PI3K-mTOR pathways: crosstalk and compensation. Trends Biochem Sci 2011;36:320-8.

[8] Omerovic J, Laude AJ, Prior IA. Ras proteins: paradigms for compartmentalised and isoform specific signalling. Cell Mol Life Sci 2007;64:2575-89.

[9] Potenza N, Vecchione C, Notte A, et al. Replacement of K-Ras with H-Ras supports normal embryonic development despite inducing cardiovascular pathology in adult mice. EMBO Rep 2005;6:432-7.

[10] Walsh AB, Bar-Sagi D. Differential activation of the Rac pathway by Ha-Ras and K-Ras. J Biol Chem 2001;1276:15609-15.

[11] Yan J, Roy S, Apolloni A, et al. Ras isoforms vary in their ability to activate Raf-1 and phosphoinositide 3-kinase. J Biol Chem 1998;273:24052-6.

[12] Seru' R, Mondola P, Damiano S, et al. Ha-Ras activates the NADPH oxidase complex in human neuroblastoma cells via extracellular signal-regulated kinase 1/2 pathway. J Neurochem 2004;91:613-22.

[13] Yang JQ Li S, Domann FE, Buettner GR, et al. Superoxide generation in v-HaRas transduced human keratinocyte $\mathrm{HaCa} \mathrm{T}$ Cells. Mol Carcinog 1999;26:180-8.

[14] Mitsushita J, Lambeth JD, Kamata T. The superoxide-generating oxidase Nox1 is functionally required for Ras oncogene transformation. Cancer Res 2004;64:3580-5.

[15] Cuda G, Paternò R, Ceravolo R. Protection of human endothelial cells from oxidative stress: role of Ras-ERK1/2 signalling. Circulation 2002;105:968-74.

[16] Recktenwald CV, Kellner R, Lichtenfels R, et al. Altered detoxification status and increased resistance to oxidative stress by K-Ras transformation. Cancer Res 2008;68:10086-93.

[17] Santillo M, Mondola P, Seru' R, et al. Opposing functions of Ki- and Ha-Ras genes in the regulation of redox signals. Curr Biol 2001;11:614-9.

[18] Wang Z, Li Y, Sarkar FH. Signalling mechanism(s) of reactive oxygen species in epithelial-mesenchymal transition reminiscent of cancer stem cells in tumour progression. Curr Stem Cell Res Ther 2010;1:74-80.

[19] Grek CL, Tew KD. Redox metabolism and malignancy. Curr Opin Pharmacol 2010;10:362-8.

[20] Radisky CD, Levy DD, Littlepage LE, et al. Rac1b and reactive oxygen species mediate MMP-3-induced EMT and genomic instability. Nature 2005;436:123-7.

[21] Cannito S, Novo E, Compagnone A, et al. Redox mechanisms switch on hypoxia-dependent epithelial-mesenchymal transition in cancer cells. Carcinogenesis 2008;29:2267-78.

[22] Wu WS. The signaling mechanism of ROS in tumor progression. Cancer Metastasis Rev 2006;25:695-705.

[23] Sadok A, Pierres A, Dahan L, et al. NADPH oxidase 1 controls the persistence of directed cell migration by a rho-dependent switch of $\alpha 2 / \alpha 3$ integrins. Mol Cell Biol 2009;29:3915-28.

[24] Ushio-Fukai M, Nakamura Y. Reactive oxygen species and angiogenesis: NADPH oxidase as target for cancer therapy. Cancer Lett 2008;266:37-52.

[25] Diaz B, Shani G, Pass I, et al. Tks5-dependent, Nox-mediated generation of reactive oxygen species is necessary for invadopodia formation. Sci Signal 2(88):ra53. http://dx.doi.org/10.1126/scisignal.2000368.

[26] Weaver AM. Regulation of cancer invasion by reactive oxygen species and Tks family scaffold proteins. Sci Signal 2(88):pe56. http://dx.doi.org/10.1126/ scisignal.288pe56.

[27] Buschman MD, Bromann PA, Cejudo-Martin P, Wen F, Pass I, Courtneidge SA. The novel adaptor protein Tks4 (SH3PXD2B) is required for functional podosome formation. Mol Biol Cell 2009;20:1302. 
[28] van Waveren C, Sun Y, Cheung HS, et al. Oxidative phosphorylation dysfunction modulates expression of extracellular matrix remodelling genes and invasion. Carcinogenesis 2006;27:409-18.

[29] Nelson KK, Melendez JA. Mitochondrial redox control of matrix metalloproteinases. Free Radical Biol Med 2004;37:768-84.

[30] Nishikawa M, Hyoudou K, Kobayashi Y, et al. Inhibition of metastatic tumour growth by targeted delivery of antioxidant enzymes. J Controlled Release 2005; 109:101-7.

[31] Shin I, Kim S, Song H, et al. H-Ras-specific activation of Rac-MKK3/6-p38 pathway. J Biol Chem 2005;280:14675-83.

[32] Moon A, Kim MS, Kim TG, et al. H-Ras, but not N-Ras, induces an invasive phenotype in human breast epithelial cells: a role for MMP-2 in the H-Rasinduced invasive phenotype. Int J Cancer 2000;85:176-81.

[33] Zhao Y, Xiao A, Dipierro CG, et al. H-Ras increases urokinase expression and cell invasion in genetically modified human astrocytes through Ras/Raf/MEK signalling pathway. Glia 2008;56:917-24.

[34] Lee KW, Kim MS, Kang NJ, et al. H-Ras selectively up-regulates MMP-9 and COX-2 through activation of ERK1/2 and NF-kB: an implication for invasive phenotype in rat liver epithelial cells. Int J Cancer 2006;119:1767-75.

[35] Campbell PM, Groehler AL, Lee KM, et al. K-Ras promotes growth transformation and invasion of immortalized human pancreatic cells by Ra and phosphatidylinositol 3-kinase signaling. Cancer Res 2007;67:2098-106.

[36] Yadav V, Denning MF. Fyn is induced by Ras/PI3K/Akt signalling and is required for enhanced invasion/migration. Mol Carcinog 2011;50:346-52.

[37] Quinlan MP, Quatela SE, Philips MR, et al. Activated K-Ras, but not H-Ras or $\mathrm{N}$-Ras, may initiate tumors of endodermal origin via stem cell expansion. $\mathrm{Mol}$ Cell Biol 2008;28:2659-74.

[38] Ahmad R, Sylvester J, Ahmad M, et al. Involvement of H-Ras and reactive oxygen species in proinflammatory cytokine-induced matrix metalloproteinase-13 expression in human articular chondrocytes. Arch Biochem Biophy 2011; 507:350-5.

[39] Yang JQ, Zhao W, Duan H, et al. V-Ha-Ras oncogene up regulates The 92-KDa type IV collagenase (Mmp-9) gene by increasing cellular superoxide production and activating NF-kB. Free Radical Biol Med 2001;31:520-9.
[40] Yang JQ, Buettner GR, Domann FE, Li J, et al. V-Ha-Ras mitogenic signaling through superoxide and derived reactive oxygen species. Mol Carcinog 2002;33:206-18

[41] Svegliati S, Cancello R, Sambo P, et al. Platelet-derived growth factor and reactive oxygen species (ROS) regulate ras protein levels in primary human fibroblasts via ERK 1/2. J Biol Chem 2005;280:36474-82.

[42] Chiarugi P. PTPs versus PTKs: the redox side of the coin. Free Radical Res 2005;39:353-64

[43] Lee K, Esselman WJ. Inhibition of PTPs by $\mathrm{H}(2) \mathrm{O}(2)$ regulates the activation of distinct MAPK pathways. Free Radical Biol Med 2002;33:1121-32.

[44] Irani K, Xia Y, Zweier JL, et al. Mitogenic signaling mediated by oxidants in Rastransformed fibroblasts. Science 1997;275:1649-51.

[45] Vilhardt F, van Deurs B. The phagocyte NADPH oxidase depends on cholesterolenriched membrane microdomains for assembly. EMBO J 2004;23:739-48.

[46] Fukano T, Sawano A, Ohba Y, et al. Differential Ras activation between caveolae/raft and non-raft microdomains. Cell Struct Funct 2007;32:9-15.

[47] Koh MS, Moon A. Activation of H-Ras and Rac1 correlates with epidermal growth factor-induced invasion in Hs578T and MDA-MB-231 breast carcinoma cells. Biochem Biophy Res Commun 2011;406:25-9.

[48] Gorzalczany Y, Sigal N, Itan M, et al. Targeting of Rac1 to the phagocyte membrane is sufficient for the induction of NADPH oxidase assembly. J Biol Chem 2000;275:40073-81.

[49] Hill BT, Perrin D, Kruczynski A. Inhibition of RAS-targeted prenylation: protein farnesyl transferase inhibitors revisited. Crit Rev Oncol/Hematol 2000;33:7-23.

[50] Yong HY, Hwang JS, Son $\mathrm{H}$, et al. Identification of H-Ras-specific motif for the activation of invasive signalling program in human breast epithelial cells. Neoplasia 2011;13:98-107.

[51] Zhang Z, Jiang G, Yang F, Wang J. Knockdown of mutant K-Ras expression by adenovirus-mediated siRNA inhibits the in vitro and in vivo growth of lung cancer cells. Cancer Biol Ther 2006;5:1481-6.

[52] Fleming JB, Shen GL, Holloway SE, et al. Molecular consequences of silencing mutant K-Ras in pancreatic cancer cells: justification for K-Ras-directed therapy. Mol Cancer Res 2005;3:413-23. 